\title{
A Survey and Comparison of License Plate Recognition using different Classifiers
}

\author{
Megha S. Utge \\ Department of Computer Engineering,MMCOE Pune, \\ University of Pune, India
}

\begin{abstract}
LPR is a well know image processing technology. Now a days vehicles play a very big role in transportation. Also the use of vehicles has been increasing because of population growth and human needs in recent years. Therefore, control of vehicles is becoming a big problem and much more difficult to solve . license plate are available in various colors and style The presence of noise, blurring in the image, uneven illumination, dim light and foggy conditions make the task even more difficult. In the proposed system ANN classifier will be used. Firstly preprocessing techniques will be done on input image, such as grayscale, blurring, thresholding and frequency calculation then segmentation. Finally use Artificial neural network classifier as a recognition method. In this paper a survey has done compared and analyzed with different recognition method.
\end{abstract}

\section{Keywords}

Plate localization ,plate detection ,Extraction, segmentation, recognition

\section{INTRODUCTION}

License plate numbers are being used to uniquely identify a vehicle LPR system plays an important role in many applications like electronic payment system (toll payment and parking fee payment), to find stolen vehicle Moreover, as increased security awareness has made the need for vehicle based authentication technologies extremely significant, the proposed system may be employed as access control system for monitoring of unauthorized vehicles entering private areas. The license plate remains as the principal vehicle identifier despite the fact that it can be deliberately altered in fraud situations or replaced. Although LPR has been an active research topic for more acknowledged. Most works are reported with performance evaluated on proprietary data sets, which are hardly available for a performance comparison. Vehicle license plate recognition is one of the key technologies to achieving the promotion of intelligent traffic management. At present, the applications of the vehicle license plate recognition technology have the following aspects: intelligent parking; urban road monitoring, traffic management, and so on. During the past few years, intelligent transportation systems (ITSs) have had a wide impact in people's life as their scope is to improve transportation safety and mobility and to enhance productivity through the use of advanced technologies

ITSs are made up of 16 types of technology based systems. These systems are divided into intelligent infrastructure systems and intelligent vehicle systems. Many methods presented recently which are based

on computer vision and character recognition algorithm for a license plate recognition (LPR) is presented to be used as a core for intelligent infrastructure like electronic payment systems (toll payment, parking fee payment), freeway, and arterial management systems for traffic surveillance.
Moreover, as increased security awareness has made the need for vehicle based authentication technologies extremely significant, the proposed system may be employed as access control system for monitoring of unauthorized vehicles entering private areas. The license plate remains as the principal vehicle identifier despite the fact that it can be deliberately altered in fraud situations or replaced (e.g., with a stolen plate). Therefore, ITSs rely heavily on robust LPR systems. The focus of this paper is on the integration of a novel segmentation technique implemented in an LPR system able to cope with outdoor conditions if parameterized properly.

License Plate Recognition System (LPRS) is a challenging problem in the field of machine vision and automation with various applications including law enforcement, parking lot ticketing systems, automated hands free toll collection, automated vehicle access in secure establishments

\section{LITERATURE SURVEY}

In the literature survey we are going to discuss ApplicationOriented License Plate Recognition: Below in literature we are discussing some of them.

C. Anagnostopoulos, I. Anagnostopoulos, V. Loumos, and E. Kayafas, "A license plate-recognition algorithm for intelligent transportation system applications," (2006) [1], In this paper, a new algorithm for vehicle license plate identification is proposed, on the basis of a novel adaptive image segmentation technique (sliding concentric windows) and connected component analysis in conjunction with a character recognition neural network. The algorithm was tested with 1334 natural-scene gray-level vehicle images of different backgrounds and ambient illumination. The camera focused in the plate, while the angle of view and the distance from the vehicle varied according to the experimental setup. The license plates properly segmented were 1287 over 1334 input images $(96.5 \%)$. The optical character recognition system is a two-layer probabilistic neural network (PNN) with topology 108-180-36, whose performance for entire plate recognition reached $89.1 \%$. The PNN is trained to identify alphanumeric characters from car license plates based on data obtained from algorithmic image processing. Combining the above two rates, the overall rate of success for the license-platerecognition algorithm is $86.0 \%$. A review in the related literature presented in this paper reveals that better performance (90\% up to $95 \%$ ) has been reported, when limitations in distance, angle of view, illumination conditions are set, and background complexity is low.

J.M. Guo and Y.F. Liu, "License plate localization and character segmentation with feedback self-learning and hybrid binarization techniques," (2008) [2], License plate localization (LPL) and character segmentation (CS) play key roles in the license plate (LP) recognition system. In this paper, we dedicate ourselves to these two issues. In LPL, histogram equalization is employed to solve the low-contrast and dynamic-range problems; the texture properties, e.g., aspect ratio, and color similarity are used to locate the LP; and the 
Hough transform is adopted to correct the rotation problem. In $\mathrm{CS}$, the hybrid binarization technique is proposed to effectively segment the characters in the dirt LP. The feedback self-learning procedure is also employed to adjust the parameters in the system. As documented in the experiments, good localization and segmentation results are achieved with the proposed algorithms.

K. Suresh, G. Kumar, and A. Rajagopalan, "Super resolution of license plates in real traffic videos," (2009) [3], In this paper, a novel method to enhance license plate numbers of moving vehicles in real traffic videos is proposed. A highresolution image of the number plate is obtained by fusing the information derived from multiple, sub pixel shifted, and noisy low-resolution observations. The image to be super resolved is modeled as a Markov random field and is estimated from the observations by a graduated non-convexity optimization procedure. A discontinuity adaptive regularize is used to preserve the edges in the reconstructed number plate for improved readability. Experimental results are given on several traffic sequences to demonstrate the robustness of the proposed method to potential errors in motion and blur estimates. The method is computationally efficient as all operations can be implemented locally in the image domain.

S.-L. Chang, L.-S. Chen, Y.-C. Chung, and S.-W. Chen, "Automatic license plate recognition," (2004) [4], preprocessing is performed to detect the Region of Interest (ROI) even in the ambient illumination conditions. It is done using image masking, binarization with sauvola method. In sauvola method, locally adaptive thresholding is used to convert a gray scale image to a binary image. The value of threshold mainly depends on the local statistics like range, variance and surface fitting parameters. In the case of badly illuminated areas, calculated threshold value will be low.

H. Caner, H. Gecim, and A. Alkar, "Efficient embedded neural-network based license plate recognition system," (2008) [5], In this paper, a video processing methodology for a field-programmable gate array (FPGA)-based license plate recognition (LPR) system is researched. The raster scan video is used as an input with low memory utilization. During the design, Gabor filter, threshold, and connected component labeling (CCL) algorithms are used to obtain license plate region. This region is segmented into disjoint characters for the character recognition phase, where the self-organizing map (SOM) neural network is used to identify the characters. The system is portable and relatively faster than computerbased recognition systems. The robustness of the system has been tested with a large database acquired from parking lots and a highway. The memory requirements are uniquely designed to be extremely low, which enables usage of smaller FPGAs. The resulting hardware is suitable for applications where cost, compactness, and efficiency are system design constraints.

In [6], C.-N. Anagnostopoulos, I. Anagnostopoulos, I. Psoroulas, V. Loumos, and E. Kayafas, "License plate recognition from still images and video sequences: A survey," a global threshold value is chosen instead of an adaptive one. To minimize the processing time, the original image is down sampled to 120 columns by preserving the original aspect ratio

G.Sun et al., in [7] divided the pre-processing task into luminance adjustment and image enhancement.

According to T.Duan et.al., [8], pre-processing is performed to enrich the edge features. The algorithms used at this stage are graying, normalizing and histogram equalization.

. In [9], improved Bernsen algorithm is used to effectively remove the shadows in the image by converting it into a binary image. In [10], image de-noising is applied to remove noises in the images. In this procedure, subtle fractures can be linked and tiny abrupt changes can be softened. The main aim of this technique is to prevent the destruction of image edges, to retain the image outline and lines as much as possible, increasing the contrast between the ROI and other regions A bi-layer classifier, which is improved with an additional null class, is experimentally proven to be better than previous methods for character recognition[11].

\section{IMPLEMENTATION DETAILS}

The proposed algorithm is drawn figure1 which consist of three main module Detection, Segmentation, Recognisation,

1.Our approach considers a input image of a vehicle where its RGB is 24 bit, to which gray scale is applied and now it is 8 bit then blurring is done to reduce the noise and disturbance

2.Apply thresholding algorithm is used to Binarize the gray scale image and generate binarized image as an output .calculate the frequency for finding the number plate area by marking starting and end point by this localization is done then cropping is applied to extact the license plate area

3. Character segmentation is done to the extracted license plate. Stentiford Thinning Algorithm is applied to thin the character then scaling, template generation, then we apply Artificial neural network for recognizing the characters.

4.An artificial neural network is composed of many artificial neurons that are linked together according to a specific network architecture. The objective of the neural network is to transform the inputs into meaningful outputs

5.ANN has two part Detection and Training, Once a network has been structured for a particular application, that network is ready to be trained. To start this process the initial weights are chosen randomly. Then, the training, or learning, begins. There are two approaches of training - supervised and unsupervised.

(a).Supervised training involves a mechanism of providing the network with the desired output either by manually "grading" the network's performance or by providing the desired outputs with the inputs.

(b).Unsupervised training is where the network has to make sense of the inputs without outside help. In supervised training, both the inputs and the outputs are provided. The network then processes the inputs and compares its resulting outputs against the desired outputs.

In unsupervised training, the network is provided with inputs but not with desired outputs. The system itself must then decide what features it will use to group the input data. This is often referred to as self-organization or adaption. 


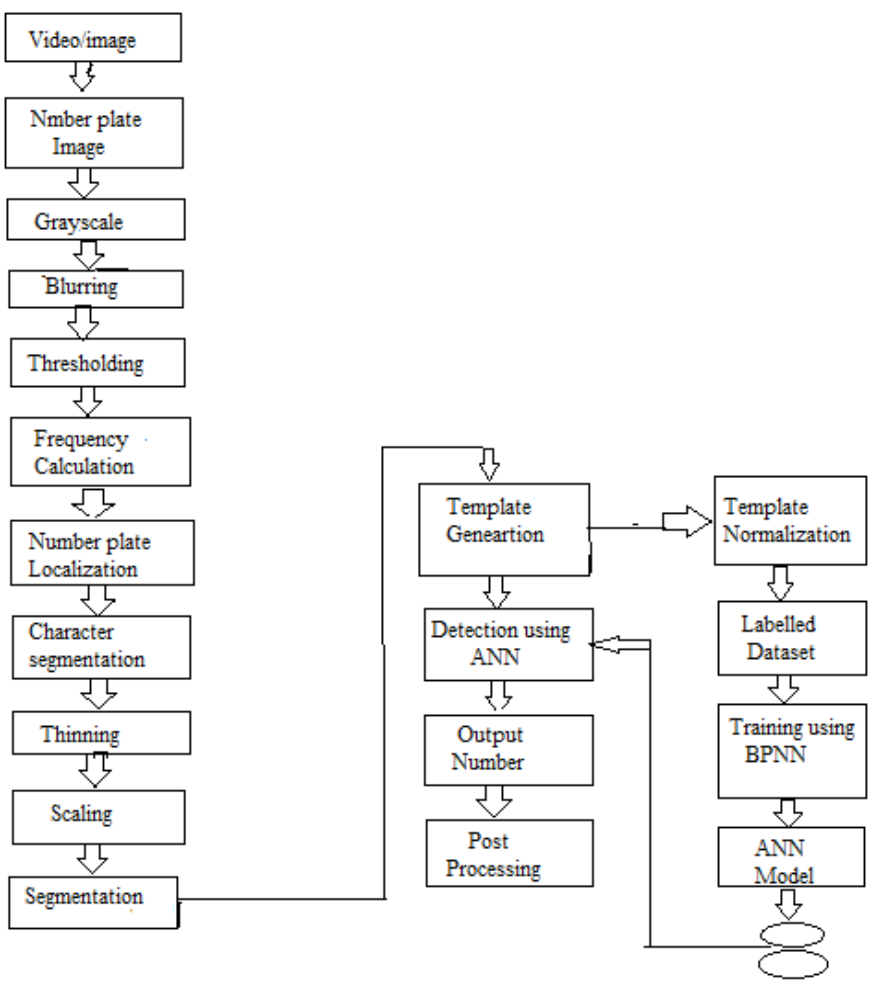

Fig1.Prosposed Architecture and Design

\section{CONCLUSION}

In this paper comparison and analysis of different LPR systems in terms of their recognition is done and discussed different problems faced by other classifiers .then introduced ANN to overcome the problems. The future work will be on working of ANN and Algorithms. The objective of this paper is to review about different LPR systems. Some LPR systems can work under uncontrolled environment but some in restricted environment. License plate recognition system mainly consists of four steps such as vehicle image capture, license plate detection, character segmentation and character recognition. From the papers surveyed, it is realized that there are different methods and algorithms used for license plate detection, character segmentation and character recognition.

\section{REFERENCES}

[1] Gee-Sern Hsu, Member, IEEE, Jiun-Chang Chen, and $\mathrm{Yu}-\mathrm{Zu}$ Chung, "Application-Oriented License Plate Recognition", IEEE TRANSACTIONS ON VEHICULAR TECHNOLOGY, VOL. 62, NO. 2, FEBRUARY 2013.

[2] J. B. Jiao, Q. X. Ye, and Q. M. Huang, "A configurable method for multistyle license plate recognition," Pattern Recognit., vol. 42, no. 3, pp. 358-369, Mar. 2009.

[3] H. Caner, H. S. Gecim, and A. Z. Alkar, "Efficient embedded neural network- based license plate recognition system," IEEE Trans. Veh. Technol., vol. 57, no. 5, pp. 2675-2683, Sep. 2008.

[4] C. Anagnostopoulos, I. Anagnostopoulos, V. Loumos, and E. Kayafas, "A license plate-recognition algorithm for intelligent transportation system applications," IEEE Trans. Intell. Transp. Syst., vol. 7, no. 3, pp. 377-392, Sep. 2006.

[5] S. L. Chang, L. S. Chen, Y. C. Chung, and S.W. Chen,
“Automatic license plate recognition,” IEEE Trans. Intell. Transp. Syst., vol. 5, no. 1, pp. 42- 52, Mar. 2004.

[6] V. Shapiro, G. Gluhchev, and D. Dimov, "Towards a multinational car license plate recognition system," Mach. Vis. Appl., vol. 17, no. 3, pp. 173-183, Aug. 2006.

[7] G.Sun, G.Li, L.Xu and J.Wangs, "The Location and Recognition of Chinese Vehicle License Plates under Complex Backgrounds," Journal of Multimedia., vol. 4, no.6, pp.442-449 Dec 2009.

[8] L.jin, H.Xian, j.Bie, Y.Sun, H.Hou "Building license plate recognition systems", Sensors 2012, vol.12, June 2012.

[9] Y.Wen, Y.Lu, J.Yan, Z.Zhou, K.M.von Deneen, and P.Shi, "An Algorithm for license Plate Recognition Applied to intelligent Transportation system," IEEE Trans.Intell.Transp.Syst., vol. 12, no.3, pp. 830-845, Sept2011.

[10] T.D.Duan, T.L.Houng, T.V.Phuoc, N.V.Hoang "License Plate Recognition Algorithm for Passenger Cars in Chinese Residential Areas", Int.Conf of Computer Science, Feb-2005

[11] 'Gee-Sern Hsu, Member, IEEE, Jiun-Chang Chen, and Yu-Zuss Chung, Application-Oriented License Plate Recognition, IEEE, VOL. 62, NO. 2, FEBRUARY 2013. 